\title{
Modulation of oxidative stress and TNF $\alpha$ secretion by peritoneal macrophages of arthritic rats fed with a flavonoid-enriched diet
}

\author{
S Ramos-Romero, F J Pérez-Cano, C Ramírez-Santana, C Castellote, A Franch, M Castell* \\ From 5th European Workshop on Immune-Mediated Inflammatory Diseases \\ Sitges-Barcelona, Spain. 1-3 December 2010
}

\section{Introduction}

The inflammatory response increases the oxidative stress and cytokine production, such as TNF $\alpha$, in macrophages. Flavonoids, present in vegetables, have potential antioxidant and anti-inflammatory properties. Previous studies have shown that a flavonoid-enriched cocoa extract added to macrophages in vitro decreases the secretion of $\mathrm{NO}$ and TNFa [1].

\section{Aim}

The objective of the present study was to ascertain the production of oxidant products and a pro-inflammatory cytokine (TNF $\alpha$ ) by peritoneal macrophages obtained from arthritic rats after a long-term cocoa diet.

\section{Methods}

Female LOU rats were fed with a $10 \%$ cocoa diet or standard chow. After 2 weeks of diet, collagen-induced arthritis (CIA) was induced in part of animals from each group. One month later, peritoneal macrophages were obtained by injecting ice-cold sterile PBS into the peritoneal cavity. Cells were cultured and stimulated by addition of $1 \mu \mathrm{g} / \mathrm{mL}$ LPS. TNF $\alpha$ secretion was quantified by ELISA in $24 \mathrm{~h}$ supernatants. In the same samples, $\mathrm{NO}_{2}{ }^{-}$concentration (stable end product of $\mathrm{NO}$ ) was measured by a modification of Griess reaction. To determine intracellular ROS production, macrophages were incubated with reduced 2',7'-dichlorofluorescein diacetate $\left(\mathrm{H}_{2} \mathrm{DCF}-\mathrm{DA}\right)$ for $30 \mathrm{~min}$ at $37{ }^{\circ} \mathrm{C}$. Fluorescence of DCF oxidized by ROS was measured by fluorometry every $30 \mathrm{~min}$ up to $2 \mathrm{~h}$.

\section{Results}

Macrophages from CIA animals fed cocoa decreased TNF $\alpha$ and NO production by $\sim 57 \%$ and $\sim 36 \%$ with respect to those from CIA reference group under LPSstimulation conditions $(\mathrm{P}<0.05)$. ROS production by macrophages from CIA animals fed standard diet was higher with respect to healthy reference animals $(\mathrm{P}<0.05)$. Cocoa diet avoided ROS secretion increase in CIA animals $(\mathrm{P}<0.05)$, raising similar values to healthy animals.

\section{Conclusion}

A long-term cocoa diet reduced the production of oxidants and TNF $\alpha$ in peritoneal macrophages obtained from collagen-induced arthritic rats.

\section{Published: 25 November 2010}

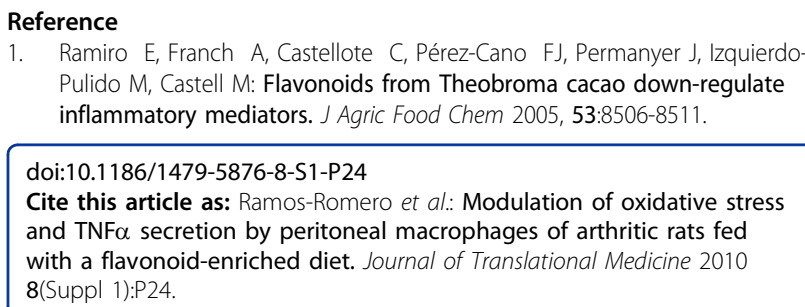

